\title{
SELF-SIMILAR RANDOM FRACTAL MEASURES USING CONTRACTION METHOD IN PROBABILISTIC METRIC SPACES
}

\author{
JÓZSEF KOLUMBÁN, ANNA SOÓS, and IBOLYA VARGA
}

Received 2 January 2003

\begin{abstract}
Self-similar random fractal measures were studied by Hutchinson and Rüschendorf. Working with probability metric in complete metric spaces, they need the first moment condition for the existence and uniqueness of these measures. In this paper, we use contraction method in probabilistic metric spaces to prove the existence and uniqueness of self-similar random fractal measures replacing the first moment condition.
\end{abstract}

2000 Mathematics Subject Classification: 60G57, 28A80.

1. Introduction. Contraction methods for proving the existence and uniqueness of nonrandom self-similar fractal sets and measures were first applied by Hutchinson [7]. Further results and applications to image compression were obtained by Barnsley and Demko [3] and Barnsley [2]. At the same time, Falconer [5], Graf [6], and Mauldin and Williams [13] randomized each step in the approximation process to obtain self-similar random fractal sets. Arbeiter [1] and Olsen [15] studied self-similar random fractal measures applying nonrandom metrics. More recently, Hutchinson and Rüschendorf [8, 9, 10] introduced probability metrics defined by expectation for random measure and established existence, uniqueness, and approximation properties of self-similar random fractal measures. In these works a finite first moment condition is essential.

In this paper, we show that, using probabilistic metric spaces techniques, we can weaken the first moment condition for the existence and uniqueness of self-similar measures.

The theory of probabilistic metric spaces, introduced in 1942 by Menger [14], was developed by numerous authors, as it can be realized upon consulting $[4,18]$ and the references therein. The study of contraction mappings for probabilistic metric spaces was initiated by Sehgal [19] and Sherwood [20].

2. Self-similar random fractal measures. Based on contraction properties of random scaling operators with respect to $l_{p}^{*}$ and $l_{p}^{* *}$, for $0<p<\infty$, on a space of random measures and their distributions, respectively, defined below, Hutchinson and Rüschendorf $[8,9,10]$ gave a simple proof for the existence and uniqueness of invariant random measures. The underlying probability 
space for the iteration procedure is also generated by selecting independent and identically distributed (i.i.d.) scaling laws for measures.

Let $(X, d)$ be a complete separable metric space.

DEFINITION 2.1. A scaling law with weights is a $2 N$-tuple

$$
\mathbf{S}:=\left(p_{1}, S_{1}, \ldots, p_{N}, S_{N}\right), \quad N \geq 1,
$$

of positive real numbers $p_{i}$ such that $\sum_{i=1}^{N} p_{i}=1$ and of Lipschitz maps $S_{i}$ : $X \rightarrow X$.

Let $r_{i}=\operatorname{Lip} S_{i}, i \in\{1, \ldots, N\}$. Denote by $M=M(X)$ the set of finite-mass Radon measures on $X$ with weak topology. If $\mu \in M$, then the measure $\mathbf{S} \mu$ is defined by

$$
\mathrm{S} \mu=\sum_{i=1}^{N} p_{i} S_{i} \mu
$$

where $S_{i} \mu$ is the usual push-forward measure, that is,

$$
S_{i} \mu(A)=\mu\left(S_{i}^{-1}(A)\right) \text { for } A \subseteq X .
$$

DEFINITION 2.2. The measure $\mu$ satisfies the scaling law $\mathbf{S}$ or is a self-similar fractal measure if $\mathrm{S} \mu=\mu$.

Let $M_{q}$ denote the set of unit mass Radon measures $\mu$ on $X$ with finite $q$ th moment; that is,

$$
M_{q}=\left\{\mu \in M \mid \mu(X)=1, \int_{X} d^{q}(x, a) d \mu(x)<\infty\right\}
$$

for some (and hence any) $a \in X$. Note that, if $p \geq q$, then $M_{p} \subset M_{q}$.

DeFinition 2.3. The minimal metric $l_{q}$ on $M_{q}$ is defined by

$$
l_{q}(\mu, v)=\inf \left\{\left(\int_{X} d^{q}(x, y) d \gamma(x, y)\right)^{1 / q \wedge 1} \mid \pi_{1} \gamma=\mu, \pi_{2} \gamma=v\right\},
$$

where $\wedge$ denotes the minimum of the relevant numbers and $\pi_{i} \gamma$ denotes the $i$ th marginal of $\gamma$, that is, projection of the measure $\gamma$ on $X \times X$ onto the $i$ th component.

The $l_{q}$ metric has the following properties (see [16]).

(a) Suppose $\alpha$ is a positive real, $S: X \rightarrow X$ is Lipschitz, and $\vee$ denotes the maximum of the relevant numbers. Then, for $q>0$ and for measures $\mu$ and $\nu$, 
we have the following properties:

$$
\begin{aligned}
l_{q}^{q \vee 1}(\alpha \mu, \alpha \nu) & =\alpha l_{q}^{q \vee 1}(\mu, \nu), \\
l_{q}^{q \vee 1}\left(\mu_{1}+\mu_{2}, v_{1}+v_{2}\right) & \leq l_{q}^{q \vee 1}\left(\mu_{1}, \nu_{1}\right)+l_{q}^{q \vee 1}\left(\mu_{2}, \nu_{2}\right), \\
l_{q}(S \mu, S \nu) & \leq(\operatorname{Lip} S)^{q \wedge 1} l_{q}(\mu, \nu) .
\end{aligned}
$$

The first property follows from the definition by setting $\gamma=c \bar{\gamma}$, where $\bar{\gamma}$ is optimal for $(\mu, \nu)$, and the third follows by setting $\gamma=S \bar{\gamma}$. The second follows by setting $\gamma=\gamma_{1}+\gamma_{2}$, where $\gamma_{i}$ is optimal for $\left(\mu_{i}, v_{i}\right)$, and also by noting that $(a+b)^{q} \leq a^{q}+b^{q}$ if $a, b \geq 0$ and $0<q<1$.

(b) The pair $\left(M_{q}, l_{q}\right)$ is a complete separable metric space and $l_{q}\left(\mu_{n}, \mu\right) \rightarrow 0$ if and only if

(i) $\mu_{n} \rightarrow \mu$ (weak convergence),

(ii) $\int_{X} d^{q}(x, a) d \mu_{n}(x) \rightarrow \int d^{q}(x, a) d \mu(x)$ (convergence of $q$ th moments).

(c) If $\delta_{a}$ is the Dirac measure at $a \in X$, then

$$
\begin{aligned}
l_{q}\left(\mu, \mu(X) \delta_{a}\right) & =\left(\int_{X} d^{q}(x, a) d \mu(x)\right)^{1 / q \wedge 1}, \\
l_{q}\left(\delta_{a}, \delta_{b}\right) & =d^{1 \wedge q}(a, b) .
\end{aligned}
$$

Let $\mathbf{M}$ denote the set of all random measures $\mu$ with value in $M$, that is, random variables $\mu: \Omega \rightarrow M$. Let $\mathbf{M}_{q}$ denote the space of random measures $\mu: \Omega \rightarrow M_{q}$ with finite expected $q$ th moment. That is,

$$
\mathbf{M}_{q}:=\left\{\mu \in \mathbf{M} \mid \mu^{\omega}(X)=1 \text { a.s., } E_{\omega} \int_{X} d^{q}(x, a) d \mu^{\omega}(x)<\infty\right\} .
$$

The notation $E_{\omega}$ indicates that the expectation is with respect to the variable $\omega$. It follows from (2.10) that $\mu^{\omega} \in M_{q}$ a.s. Note that $\mathbf{M}_{p} \subset \mathbf{M}_{q}$ if $q \leq p$. Moreover, since $E^{1 / q}|f|^{q} \rightarrow \exp (E \log |f|)$ as $q \rightarrow 0$,

$$
\mathbf{M}_{0}:=\cup_{q>0} \mathbf{M}_{q}=\left\{\mu \in \mathbf{M} \mid \mu_{\omega}(X)=1 \text { a.s., } E_{\omega} \int_{X} \log d(x, a) d \mu^{\omega}(x)<\infty\right\} .
$$

For random measures $\mu, v \in \mathbf{M}_{q}$, define

$$
l_{q}^{*}(\mu, \nu):= \begin{cases}E_{\omega}^{1 / q} l_{q}^{q}\left(\mu^{\omega}, \nu^{\omega}\right), & q \geq 1, \\ E_{\omega} l_{q}\left(\mu^{\omega}, \nu^{\omega}\right), & 0<q<1 .\end{cases}
$$

One can check, as in [16], that $\left(\mathbf{M}_{q}, \mathbf{l}_{q}^{*}\right)$ is a complete separable metric space. Note that $l_{q}^{*}(\mu, \nu)=l_{q}(\mu, v)$ if $\mu$ and $v$ are constant random measures. 
Let $\boldsymbol{M}$ denote the class of probability distributions on $\mathbf{M}$, that is,

$$
\mathcal{M}=\{\mathscr{D}=\operatorname{dist} \mu \mid \mu \in \mathbf{M}\} .
$$

Let $\mu_{q}$ be the set of probability distributions of random measures $\mu \in \mathbf{M}_{q}$. For $q \leq p$, it is to be noticed that $\mu_{p} \subset M_{q}$. Let

$$
M_{0}:=\cup_{q>0} M_{q}
$$

The minimal metric on $\mu_{q}$ is defined by

$$
l_{q}^{* *}\left(\mathscr{D}_{1}, \mathscr{D}_{2}\right)=\inf \left\{l_{q}^{*}(\mu, v) \mid \mu \stackrel{d}{=} D_{1}, \nu \stackrel{d}{=} D_{2}\right\} .
$$

It follows that $\left(M_{q}, l_{q}^{* *}\right)$ is a complete separable metric space with the next properties (see [16]):

(a) $l_{q}^{* *}\left(\alpha \mathscr{D}_{1}, \alpha \mathscr{D}_{2}\right)=\alpha l_{q}^{* *}\left(\mathscr{D}_{1}, \mathscr{D}_{2}\right)$,

(b) $l_{q}^{* *}\left(\mathscr{D}_{1}+\mathscr{D}_{2}, \mathscr{D}_{3}+\mathscr{D}_{4}\right) \leq l_{q}^{* * q}\left(\mathscr{D}_{1}, \mathscr{D}_{3}\right)+l_{q}^{* * q}\left(\mathscr{D}_{2}, \mathscr{D}_{4}\right)$, for $\mathscr{D}_{i} \in \mathcal{M}_{q}, i=1,2,3,4$.

DEFINITION 2.4. A random scaling law with weights or a random scaling law for measure $\mathbb{S}=\left(p_{1}, S_{1}, p_{2}, S_{2}, \ldots, p_{N}, S_{N}\right)$ is a random variable whose values are scaling laws, with $\sum_{i=1}^{N} p_{i}=1$ a.s.

We write $\mathscr{S}=\operatorname{dist} \mathbb{S}$ for the probability distribution determined by $\mathbb{S}$.

If $\mu$ is a random measure, then the random measure $\mathbb{S} \mu$ is defined (up to probability distribution) by

$$
\mathbb{S} \mu:=\sum_{i=1}^{N} p_{i} S_{i} \mu^{(i)},
$$

where $\mathbb{S}, \mu^{(1)}, \ldots, \mu^{(N)}$ are independent of one another, and $\mu^{(i)} \stackrel{d}{=} \mu$. If $\mathscr{D}=$ dist $\mu$, we define $\mathscr{S} \mathscr{D}=\operatorname{dist} \mathbb{S} \mu$.

DEFINITION 2.5. The measure $\mu$ satisfies the scaling law $\mathbb{S}$ or is a self-similar random fractal measure if $\mathbb{S} \mu \stackrel{d}{=} \mu$, or equivalently $\mathscr{\mathscr { D }}=\mathscr{D}$, where $\mathscr{D}$ is called a self-similar random fractal distribution.

To generate a random self-similar fractal measure, we use the iterative procedure described as follows. Fix $q>0$. Beginning with a nonrandom measure $\mu_{0} \in M_{q}$ (or, more generally, a random measure $\mu_{0} \in \mathbf{M}_{q}$ ), one iteratively applies i.i.d. scaling laws with distribution $\mathscr{S}$ to obtain a sequence $\mu_{n}$ of random measures in $\mathbf{M}_{q}$ and a corresponding sequence $\mathscr{D}_{n}$ of distributions in $\mu_{q}$ as follows.

(i) Select a scaling law $\mathbb{S}$ via the distribution $\mathscr{S}$ and define

$$
\mu_{1}=\mathbb{S} \mu_{0}=\sum_{i=1}^{n} p_{i} S_{i} \mu_{0},
$$


that is,

$$
\mu_{1}(\omega)=\mathbb{S} \mu_{0}=\sum_{i=1}^{n} p_{i}(\omega) S_{i}(\omega) \mu_{0}, \quad \mathscr{D}_{1} \stackrel{d}{=} \mu_{1}
$$

(ii) Select $\mathbb{S}^{1}, \ldots, \mathbb{S}^{N}$ via $\mathscr{S}$ with $\mathbb{S}^{i}=\left(p_{1}^{i}, S_{1}^{i}, \ldots, p_{N}^{i}, S_{N}^{i}\right), i \in\{1,2, \ldots, N\}$, independent of each other and of $\mathbb{S}$, and define

$$
\mu_{2}:=\mathbb{S}^{2} \mu_{0}=\sum_{i, j} p_{i} p_{j}^{i} S_{i} \circ S_{j}^{i} \mu_{0}, \quad \mathscr{D}_{2} \stackrel{d}{=} \mu_{0} .
$$

(iii) Select $\mathbb{S}^{i j}=\left(p_{1}^{i}, S_{1}^{i j}, \ldots, p_{N}^{i}, S_{N}^{i, j}\right)$ via $\mathscr{Y}$, independent of one another and of $\mathbb{S}^{1}, \ldots, \mathbb{S}^{N}, S$, and define

$$
\mu_{3}=\mathbb{S}^{3} \mu_{0}=\sum_{i, j, k} p_{i} p_{j}^{i} p_{k}^{i j} S_{i} \circ S_{j}^{i} \circ S_{k}^{i j} \mu_{0}, \quad \mathscr{D}_{3} \stackrel{d}{=} \mu_{3}
$$

and so forth.

Thus $\mu_{n+1}=\sum_{i=1}^{N} p_{i} S_{i} \mu_{n}^{(i)}$, where $\mu_{n}^{(i)} \stackrel{d}{=} \mu_{n} \stackrel{d}{=} \mathscr{D}_{n}, \mathbb{S} \stackrel{d}{=} \mathscr{Y}$, and $\mu_{n}^{(i)}$ and $\mathbf{S}$ are independent. It follows that $\mathscr{D}_{n}=\mathscr{S}_{N-1}=\mathscr{S}^{n} \mathscr{D}_{0}$, where $\mathscr{D}_{0}$ is the distribution of $\mu_{0}$. In the case $\mu_{0} \in M_{q}$, $\mathscr{D}_{0}$ is constant.

In the following, we define the underlying probability space for a.s. convergence (see [10]).

A construction tree (or a construction process) is a map $\omega:\{1, \ldots, N\}^{*} \rightarrow \Gamma$, where $\Gamma$ is the set of (nonrandom) scaling laws. A construction tree specifies, at each node of the scaling law used for constructive definition, a recursive sequence of random measures. Denote the scaling law of $\omega$ at the node $\sigma$ by the $2 N$-tuple

$$
\mathbf{S}^{\sigma}(\omega)=\omega(\sigma)=\left(p_{1}^{\sigma}(\omega), S_{1}^{\sigma}(\omega), \ldots, p_{N}^{\sigma}(\omega), S_{N}^{\sigma}(\omega)\right)
$$

where $p_{i}^{\sigma}$ are weights and $S_{i}^{\sigma}$ Lipschitz maps. The sample space of all construction trees is denoted by $\tilde{\Omega}$. The underlying probability space $(\tilde{\Omega}, \tilde{\mathscr{K}}, \tilde{P})$ for the iteration procedure is generated by selecting i.i.d. scaling laws $\omega(\sigma) \stackrel{d}{=} \mathbf{S}$ for each $\sigma \in\{1, \ldots, N\}^{*}$. We use the notation

$$
\begin{aligned}
& \bar{p}^{\sigma}=p_{\sigma_{1}} p_{\sigma_{2}}^{\sigma_{1}} p_{\sigma_{3}}^{\sigma_{1} \sigma_{2}} \cdots p_{\sigma_{n}}^{\sigma_{1} \cdots \sigma_{n-1}}, \\
& \bar{S}^{\sigma}=S_{\sigma_{1}} S_{\sigma_{2}}^{\sigma_{1}} p_{\sigma_{3}}^{\sigma_{1} \sigma_{2}} \cdots S_{\sigma_{n}}^{\sigma_{1} \cdots \sigma_{n-1}},
\end{aligned}
$$

where $|\sigma|=n$ and where $p_{i}^{\sigma}$ and $S_{i}^{\sigma}$ denote the $i$ th components of scaling law. For a fixed measure $\mu_{0} \in M_{\mathfrak{q}}$, define

$$
\mu_{n}=\mu_{n}(\omega)=\sum_{|\sigma|=n} \bar{p}^{\sigma}(\omega) \bar{S}^{\sigma}(\omega) \mu_{0}
$$


for $n \geq 1$. This is identical to the sequence defined in an iterative procedure with an underlying space $\Omega=\bar{\Omega}$. To see this, for $\omega \in \bar{\Omega}$ and $1 \leq i \leq N$, let $\omega^{(i)} \in \bar{\Omega}$ be defined by

$$
\omega^{(i)}(\sigma)=\omega(i * \sigma)
$$

for $\sigma \in\{1, \ldots, N\}^{*}$. Then

$$
\begin{aligned}
& \bar{p}^{i * \sigma}=p_{i}(\omega) \bar{p}^{\sigma}\left(\omega^{(i)}\right), \\
& \bar{S}^{i * \sigma}=S_{i}(\omega) \circ \bar{p}^{\sigma}\left(\omega^{(i)}\right) .
\end{aligned}
$$

By construction, $\omega^{(i)}$ are i.i.d. with the same distribution as $\omega$, and are independent of $\left(p_{1}(\omega), S_{1}(\omega), \ldots, p_{N}(\omega), S_{N}(\omega)\right)$. More precisely, for any $\bar{P}$ measurable sets $E, F \subset \bar{\Omega}$ and $B \subset \Gamma$,

$$
\bar{P}(\{\omega \mid \omega \in E\})=\bar{P}\left(\left\{\omega \mid \omega^{(i)} \in E\right\}\right),
$$

where $\left\{\omega \mid \omega^{(i)} \in E\right\}$ and $\left\{\omega \mid \omega^{(j)} \in E\right\}$ are independent if $i \neq j$, and $\{\omega \mid$ $\left.\left(p_{1} \omega, S_{1}(\omega), \ldots, p_{N}(\omega), S_{N}(\omega)\right) \in B\right\}$ and $\left\{\omega \mid \omega^{(i)} \in E\right\}$ are independent. It follows that

$$
\mu_{n+1}(\omega)=\sum_{i=1}^{N} \sum_{|\sigma|=n} \bar{p}^{i * \sigma} \bar{S}^{i * \sigma}(\omega) \mu_{0}=\sum_{i=1}^{N} p_{i}(\omega) S_{i}(\omega) \mu_{n}\left(\omega^{(i)}\right)=\mathbf{S} \mu_{n}(\omega) .
$$

In [8], Hutchinson and Rüschendorf proved the following theorem.

THEOREM 2.6. Let $\mathbb{S}=\left(p_{1}, S_{1}, p_{2} S_{2}, \ldots, p_{N}, S_{N}\right)$ be a random scaling law with $\sum_{i=1}^{N} p_{i}=1$ a.s. Assume $\lambda_{q}:=E_{\omega}\left(\sum_{i=1}^{N} p_{i} r_{i}^{q}\right)<1$ and

$$
E_{\omega}\left(\sum_{i=1}^{N} p_{i} d^{q}\left(S_{i} a, a\right)\right)<\infty \text { for some } q>0, \text { and for } a \in Y
$$

Then the following facts hold.

(a) The operator $\mathbb{S}: M_{\mathcal{q}} \rightarrow M_{q}$ is a contraction map with respect to $l_{q}^{*}$.

(b) There exists a self-similar random measure $\mu^{*}$.

(c) If $\mu_{0} \in M_{p}$ (or, more generally, $\mathbf{M}_{q}$ ), then

$$
\begin{aligned}
& E_{\omega}^{1 / q} l_{q}^{q}\left(\mu_{k}, \mu^{*}\right) \leq \frac{\lambda_{q}^{k / q}}{1-\lambda_{q}^{1 / q}} E_{\omega}^{1 / q} l_{q}^{q}\left(\mu_{1}, \mathrm{~S} \mu_{0}\right) \longrightarrow 0, \quad q \geq 1, \\
& E_{\omega} l_{q}\left(\mu_{k}, \mu^{*}\right) \leq \frac{\lambda_{q}^{k}}{1-\lambda_{q}} E_{\omega} l_{q}\left(\mu_{1}, \mathrm{~S} \mu_{0}\right) \longrightarrow 0, \quad 0<q<1,
\end{aligned}
$$

as $k \rightarrow \infty$. In particular $\mu_{n} \rightarrow \mu^{*}$ a.s. in the sense of weak convergence of measures.

Moreover, up to probability distribution, $\mu^{*}$ is the unique unit mass random measure with $E_{\omega} \int \ln d(x, a) d \mu^{\omega}<\infty$, which satisfies $\mathbb{S}$. 
Using contraction method in probabilistic metric spaces, instead of condition (2.28), we can give a weaker condition for the existence and uniqueness of invariant measure. More precisely, we prove the following theorem.

THEOREM 2.7. Let $\mathbb{S}=\left(p_{1}, S_{1}, p_{2}, S_{2}, \ldots, p_{N}, S_{N}\right)$ be a random scaling law which satisfies $\sum_{i=1}^{N} p_{i}=1$ a.s., and suppose $\lambda_{q}:=\operatorname{ess} \sup \left(\sum_{i=1}^{N} p_{i} r_{i}^{q}\right)<1$ for some $q>0$. If there exist $\alpha \in M_{q}$ and a positive number $\gamma$ such that

$$
P\left(\left\{\omega \in \Omega \mid l_{q}(\alpha(\omega), \mathbb{S} \alpha(\omega)) \geq t\right\}\right) \leq \frac{\gamma}{t} \quad \forall t>0,
$$

then there exists $\mu^{*}$ such that $\mathbb{S} \mu^{*}=\mu^{*}$ a.s.

Moreover, up to probability distribution, $\mu^{*}$ is the unique unit mass random measure which satisfies $\mathbb{\$}$.

REMARK 2.8. If condition (2.28) is satisfied, then condition (2.30) also holds. To see this, let $a \in X$ and $\alpha(\omega):=\delta_{a}$ for all $\omega \in \Omega$. We have

$$
\begin{aligned}
P(\{\omega & \left.\left.\in \Omega \mid l_{q}\left(\delta_{a}(\omega), \mathbb{S} \delta_{a}(\omega)\right) \geq t\right\}\right) \\
& =P\left(\left\{\omega \in \Omega \mid l_{q}\left(\sum_{i=1}^{N} p_{i} \delta_{a}(\omega), \sum_{i=1}^{N} p_{i} S_{i} \delta_{a}(\omega)\right) \geq t\right\}\right) \\
& \leq P\left(\left\{\omega \in \Omega \mid \sum_{i=1}^{N} p_{i} l_{q}\left(\delta_{a}(\omega), S_{i} \delta_{a}(\omega)\right) \geq t\right\}\right) \\
& =P\left(\left\{\omega \in \Omega \mid \sum_{i=1}^{N} p_{i} d^{q}\left(S_{i} a, a\right) \geq t\right\}\right) \\
& \leq \frac{1}{t} E_{\omega}\left(\sum_{i=1}^{N} p_{i} d^{q}\left(S_{i} a, a\right)\right)=\frac{\gamma}{t} .
\end{aligned}
$$

However, condition (2.30) can also be satisfied if

$$
E_{\omega}\left(\sum_{i=1}^{N} p_{i} d^{q}\left(S_{i} a, a\right)\right)=\infty \quad \forall q>0 .
$$

Let $\Omega=] 0,1]$ with the Lebesque measure, let $X$ be the interval $[0, \infty[$, and let $N=1$. Define $S: X \rightarrow X$ by $S^{\omega}(x)=x / 2+e^{1 / \omega}$. This map is a contraction with ratio $1 / 2$. For $q>0$, the expectation $E_{\omega} d^{q}(S 0,0)=\infty$, however

$$
P\left(\left\{\omega \in \Omega \mid l_{q}(S 0,0) \geq t\right\}\right)=\frac{1}{t}
$$

for all $t>0$.

\section{Invariant sets in $E$-spaces}

3.1. Menger spaces. Let $\mathbb{R}$ denote the set of real numbers and $\mathbb{R}_{+}:=\{x \in$ $\mathbb{R}: x \geq 0\}$. A mapping $F: \mathbb{R} \rightarrow[0,1]$ is called a distribution function if it is 
nondecreasing, left continuous with $\inf _{t \in \mathbb{R}} F(t)=0$ and $\sup _{t \in \mathbb{R}} F(t)=1$ (see [4]). By $\Delta$ we will denote the set of all distribution functions $F$. Let $\Delta$ be ordered by the relation " $\leq$ ", that is, $F \leq G$ if and only if $F(t) \leq G(t)$ for all real $t$. Also $F<G$ if and only if $F \leq G$ but $F \neq G$. We set $\Delta^{+}:=\{F \in \Delta: F(0)=0\}$.

Throughout this paper, $H$ will denote the heaviside distribution function defined by

$$
H(x)= \begin{cases}0, & x \leq 0 \\ 1, & x>0\end{cases}
$$

Let $X$ be a nonempty set. For a mapping $\mathscr{F}: X \times X \rightarrow \Delta^{+}$and $x, y \in X$, we will denote $\mathscr{F}_{\mathcal{F}}(x, y)$ by $F_{x, y}$, and the value of $F_{x, y}$ at $t \in \mathbb{R}$ by $F_{x, y}(t)$, respectively. The pair $(X, \mathscr{F})$ is a probabilistic metric space (briefly PM space) if $X$ is a nonempty set and $\mathscr{F}: X \times X \rightarrow \Delta^{+}$is a mapping satisfying the following conditions:

(1) $F_{x, y}(t)=F_{y, x}(t)$ for all $x, y \in X$ and $t \in \mathbb{R}$;

(2) $F_{x, y}(t)=1$, for every $t>0$, if and only if $x=y$;

(3) if $F_{x, y}(s)=1$ and $F_{y, z}(t)=1$, then $F_{x, z}(s+t)=1$.

A mapping $T:[0,1] \times[0,1] \rightarrow[0,1]$ is called a $t$-norm if the following conditions are satisfied:

(4) $T(a, 1)=a$ for every $a \in[0,1]$;

(5) $T(a, b)=T(b, a)$ for every $a, b \in[0,1]$;

(6) if $a \geq c$ and $b \geq d$, then $T(a, b) \geq T(c, d)$;

(7) $T(a, T(b, c))=T(T(a, b), c)$ for every $a, b, c \in[0,1]$.

A Menger space is a triplet $(X, \mathscr{F}, T)$, where $(X, \mathscr{F})$ is a PM space, $T$ is a $t$ norm, and instead of condition (3), we have the stronger condition

(8) $F_{x, y}(s+t) \geq T\left(F_{x, z}(s), F_{z, y}(t)\right)$ for all $x, y, z \in X$ and $s, t \in \mathbb{R}_{+}$.

The $(t, \epsilon)$-topology in a Menger space was introduced in 1960 by Schweizer and Sklar [17]. The base for the neighbourhoods of an element $x \in X$ is given by

$$
\left\{U_{x}(t, \epsilon) \subseteq X: t>0, \epsilon \in\right] 0,1[\}
$$

where

$$
U_{x}(t, \epsilon):=\left\{y \in X: F_{x, y}(t)>1-\epsilon\right\}
$$

In 1969, Sehgal [19] introduced the notion of a contraction mapping in PM spaces. The mapping $f: X \rightarrow X$ is said to be a contraction if there exists $r \in$ ] $0,1[$ such that

$$
F_{f(x), f(y)}(r t) \geq F_{x, y}(t)
$$

for every $x, y \in X$ and $t \in \mathbb{R}_{+}$. 
A sequence $\left(x_{n}\right)_{n \in \mathbb{N}}$ from $X$ is said to be fundamental if

$$
\lim _{n, m \rightarrow \infty} F_{x_{m}, x_{n}}(t)=1
$$

for all $t>0$. The element $x \in X$ is called limit of the sequence $\left(x_{n}\right)_{n \in \mathbb{N}}$, and we write $\lim _{n \rightarrow \infty} x_{n}=x$ or $x_{n} \rightarrow x$ if $\lim _{n \rightarrow \infty} F_{x, x_{n}}(t)=1$ for all $t>0$. A PM (Menger) space is said to be complete if every fundamental sequence in that space is convergent.

Let $A$ and $B$ be nonempty subsets of $X$. The probabilistic Hausdorff-Pompeiu distance between $A$ and $B$ is the function $F_{A, B}: \mathbb{R} \rightarrow[0,1]$ defined by

$$
F_{A, B}(t):=\sup _{s<t} T\left(\inf _{x \in A} \sup _{y \in B} F_{x, y}(s), \inf _{y \in B} \sup _{x \in A} F_{x, y}(s)\right) .
$$

In the following, we recall some properties proved in [11, 12].

Proposition 3.1. If $\mathscr{b}$ is a nonempty collection of nonempty closed bounded sets in a Menger space $(X, \mathscr{F}, T)$ with $T$ continuous, then $\left(\mathscr{C}_{,}, \mathscr{F}_{\mathscr{C}}, T\right)$ is also Menger space, where $\mathscr{F}_{\mathscr{C}}$ is defined by $\mathscr{F}_{\mathscr{C}}(A, B):=F_{A, B}$ for all $A, B \in \mathscr{C}$.

Proposition 3.2. Let $T_{m}(a, b):=\max \{a+b-1,0\}$. If $\left(X, \mathscr{F}, T_{m}\right)$ is a complete Menger space and $\mathscr{C}$ is the collection of all nonempty closed bounded subsets of $X$ in $(t, \epsilon)$-topology, then $\left(\mathscr{C}, \mathscr{F}_{\mathscr{C}}, T_{m}\right)$ is also a complete Menger space.

3.2. $E$-spaces. The notion of $E$-space was introduced by Sherwood [20] in 1969 . Next we recall this definition. Let $(\Omega, \mathscr{K}, P)$ be a probability space and let $(Y, \rho)$ be a metric space. The ordered pair $(\mathscr{E}, \mathscr{F})$ is an $E$-space over the metric space $(Y, \rho)$ (briefly, an $E$-space) if the elements of $\mathscr{E}$ are random variables from $\Omega$ into $Y$ and $\mathscr{F}$ is the mapping from $\mathscr{E} \times \mathscr{E}$ into $\Delta^{+}$defined via $\mathscr{F}(x, y)=F_{x, y}$, where

$$
F_{x, y}(t)=P(\{\omega \in \Omega \mid d(x(\omega), y(\omega))<t\})
$$

for every $t \in \mathbb{R}$. Usually $(\Omega, \mathscr{K}, P)$ is called the base and $(Y, \rho)$ the target space of the $E$-space. If $\mathscr{F}$ satisfies the condition

$$
\mathscr{F}(x, y) \neq H \text { for } x \neq y \text {, }
$$

with $H$ defined in Section 3.1, then $(\mathscr{E}, \mathscr{F})$ is said to be a canonical E-space. Sherwood [20] proved that every canonical $E$-space is a Menger space under $T=T_{m}$, where $T_{m}(a, b)=\max \{a+b-1,0\}$. In the following, we suppose that $\mathscr{E}$ is a canonical $E$-space.

The convergence in an $E$-space is exactly the probability convergence. The $E$-space $(\mathscr{E}, \mathscr{F})$ is said to be complete if the Menger space $\left(\mathscr{E}, \mathscr{F}, T_{m}\right)$ is complete.

Proposition 3.3. If $(Y, \rho)$ is a complete metric space, then the E-space $(\mathscr{E}, \mathscr{F})$ is also complete. 
Proof. This property is well known for $Y=R$ (see, e.g., [21, Theorem VII.4.2]). In the general case, the proof is analogous.

Let $\left(x_{n}\right)_{n \in \mathbb{N}}$ be a Cauchy sequence of elements of $\mathscr{E}$, that is,

$$
\lim _{n, m \rightarrow \infty} F_{x_{n}, x_{n+m}}(t)=1 \quad \forall t>0 .
$$

First we show that there exists a subsequence $\left(x_{n_{k}}\right)_{k \in \mathbb{N}}$ of the given sequence which is convergent almost everywhere to a random variable $x$. We set positive numbers $\epsilon_{i}$ so that $\sum_{i=1}^{\infty} \epsilon_{i}<\infty$ and put $\delta_{p}=\sum_{i=p}^{\infty} \epsilon_{i}, p=1,2, \ldots$. For each $i$, there is a natural number $k_{i}$ such that

$$
P\left(\left\{\omega \in \Omega \mid \rho\left(x_{k}(\omega), x_{l}(\omega)\right) \geq \epsilon_{i}\right\}\right)<\epsilon_{i} \text { for } k, l \geq k_{i}
$$

We can assume that $k_{1}<k_{2}<\cdots<k_{i}<\cdots$. Then

$$
P\left(\left\{\omega \in \Omega \mid \rho\left(x_{k_{i+1}}(\omega), x_{k_{i}}(\omega)\right) \geq \epsilon_{i}\right\}\right)<\epsilon_{i} \text { for } k, l \geq k_{i} .
$$

We put

$$
D_{p}=\cup_{i=p}^{\infty}\left\{\omega \in \Omega \mid \rho\left(x_{k_{i+1}}, x_{k_{i}}\right) \geq \epsilon_{i}\right\}
$$

Then $P\left(D_{p}\right)<\delta_{p}$. Finally, for the intersection $D^{\prime}=\cap_{p=1}^{\infty} D_{p}$, we obviously have $P\left(D^{\prime}\right)=0$ since $\delta_{p} \rightarrow 0$. We will show that the sequence $\left(x_{k_{i}}(\omega)\right)$ has a finite limit $x(\omega)$ at every point $\omega \in\left\{\omega \in \Omega \mid \rho\left(x_{k}(\omega), x_{m}(\omega)\right)>t\right\} \backslash D^{\prime}$. For some $p$ we have $x \notin D_{p}$. Consequently, $\rho\left(x_{k_{i+1}}(\omega), x_{k_{i}}(\omega)\right)<\epsilon_{i}$, for all $i \geq p$. It follows that for any two indices $i$ and $j$ such that $j>i \geq p$, we have

$$
\rho\left(x_{k_{j}}(\omega), x_{k_{i}}(\omega)\right) \leq \sum_{m=i}^{j-1} \rho\left(x_{k_{m+1}}(\omega), x_{k_{m}}(\omega)\right)<\sum_{m=i}^{j-1} \epsilon_{m}<\sum_{m=i}^{\infty} \epsilon_{m}=\delta_{i} .
$$

Thus $\lim _{i, j \rightarrow \infty} \rho\left(x_{k_{j}}(\omega), x_{k_{i}}(\omega)\right)=0$. This means that $\left(x_{k}(\omega)\right)_{k \in \mathbb{N}}$ is a Cauchy sequence for every $\omega$ which implies the pointwise convergence of $\left(x_{k_{i}}\right)_{i \in \mathbb{N}}$ to a finite-limit function. Now remains only to put

$$
x(\omega)= \begin{cases}\lim x_{k_{i}}(\omega) & \text { for } \omega \notin D^{\prime} \\ 0 & \text { for } \omega \in D^{\prime}\end{cases}
$$

to obtain the desired limit random variable. By Lebesque theorem (see, e.g., [21, Theorem VI.5.2]), $x_{k_{i}} \rightarrow x$ with respect to $d$. Thus, every Cauchy sequence in $\mathscr{E}$ has a limit, which means that the space $\mathscr{E}$ is complete.

The next result was proved in [12]. 
THEOREM 3.4. Let $(\mathscr{E}, \mathscr{F})$ be a complete E-space, $N \in \mathbb{N}^{*}$, and let $f_{1}, \ldots, f_{N}$ : $\mathscr{E} \rightarrow \mathscr{E}$ be contractions with ratios $r_{1}, \ldots, r_{N}$, respectively. Suppose that there exist an element $z \in \mathscr{E}$ and a real number $\gamma$ such that

$$
P\left(\left\{\omega \in \Omega \mid \rho\left(z(\omega), f_{i}(z(\omega))\right) \geq t\right\}\right) \leq \frac{\gamma}{t}
$$

for all $i \in\{1, \ldots, N\}$ and for all $t>0$. Then there exists a unique nonempty closed bounded and compact subset $K$ of $\mathscr{E}$ such that

$$
f_{1}(K) \cup \cdots \cup f_{N}(K)=K .
$$

COROLlary 3.5. Let $(\mathscr{E}, \mathscr{F})$ be a complete E-space and let $f: \mathscr{E} \rightarrow \mathscr{E}$ be a contraction with ratio $r$. Suppose there exist $z \in \mathscr{E}$ and a real number $\gamma$ such that

$$
P(\{\omega \in \Omega \mid \rho(z(\omega), f(z)(\omega)) \geq t\}) \leq \frac{\gamma}{t} \quad \forall t>0 .
$$

Then there exists a unique $x_{0} \in \mathscr{E}$ such that $f\left(x_{0}\right)=x_{0}$.

4. Proof of Theorem 2.7. Before the proof of the theorem, we give two lemmas.

Let $\mathscr{E}_{q}$ be the set of random variables with values in $M_{q}$ and let $\mathscr{E}_{q}(\alpha)$ be the set

$$
\mathscr{E}_{q}(\alpha):=\left\{\beta \in \mathscr{E}_{q} \mid \exists \gamma>0, P\left(\left\{\omega \in \Omega \mid l_{q}(\alpha(\omega), \beta(\omega)) \geq t\right\}\right) \leq \frac{\gamma}{t} \forall t>0\right\} .
$$

LEMMA 4.1. For all $\alpha \in M_{q}, \mathbf{M}_{q} \subset \mathscr{E}_{q}(\alpha)$.

Proof. For $\beta \in \mathbf{M}_{q}$, we have

$$
\begin{aligned}
& P\left(\left\{\omega \in \Omega \mid l_{q}(\alpha(\omega), \beta(\omega)) \geq t\right\}\right) \\
& \quad=\int_{l_{q}(\alpha(\omega), \beta(\omega)) \geq t} d P \leq \frac{1}{t} \int_{\Omega} l_{q}(\alpha(\omega), \beta(\omega)) d P=\frac{1}{t} E_{\omega} l_{q}(\alpha(\omega), \beta(\omega)) .
\end{aligned}
$$

Since $\beta \in M_{q}$, we have $\gamma=E_{\omega} l_{q}(\alpha(\omega), \beta(\omega))<\infty$ for all $t>0$.

LEMMA 4.2. The pair $\left(\mathscr{E}_{q}, \mathscr{F}\right)$ is a complete E-space.

Proof. The lemma follows by choosing $Y:=\mathscr{E}_{q}$ and $\mathscr{F}_{\mu, v}(t):=P(\{\omega \in \Omega \mid$ $\left.\left.l_{q}(\mu(\omega), v(\omega))<t\right\}\right)$ in Proposition 3.3.

Proof of Theorem 2.7. Let $\mathbb{S}$ be a random scaling law. Define $f: \mathscr{E}_{q} \rightarrow \mathscr{E}_{q}$ by $f(\mu)=\mathbf{S} \mu$, that is,

$$
\mathrm{S} \mu(\omega)=\sum_{i} p_{i}^{\omega} S_{i}^{\omega} \mu\left(\omega^{(i)}\right)
$$


We first claim that if $\mu \in \mathscr{E}_{q}$, then $\mathbb{S} \mu \in \mathscr{E}_{q}$. For this, choose i.i.d. $\mu\left(\omega^{(i)}\right) \stackrel{d}{=} \mu(\omega)$ and $\left(p_{1}^{\omega}, S_{1}^{\omega}, \ldots, p_{N}^{\omega}, S_{N}^{\omega}\right) \stackrel{d}{=} \mathbb{S}$ independent of $\mu(\omega)$. For $q \geq 1$ and $b_{i}=S_{i}^{-1}(a)$, using (2.8), we compute

$$
\begin{aligned}
\int_{X} d^{q}(x, a) d\left(\mathbb{S} \mu^{\omega}(x)\right) & =l_{q}^{q}\left(\sum_{i=1}^{N} p_{i}^{\omega} S_{i}^{\omega} \mu\left(\omega^{(i)}\right), \delta_{a}\right) \\
& =l_{q}^{q}\left(\sum_{i=1}^{N} p_{i}^{\omega} S_{i}^{\omega} \mu\left(\omega^{(i)}\right), \sum_{i=1}^{N} p_{i}^{\omega} S_{i}^{\omega} \delta_{b_{i}}\right) \\
& \leq \sum_{i=1}^{N} p_{i}^{\omega} r_{i}^{q} l_{q}^{q}\left(\mu\left(\omega^{(i)}\right), \delta_{b_{i}}\right) .
\end{aligned}
$$

Since $\mu\left(\omega^{(i)}\right) \in M_{q}$, we have

$$
\int_{X} d^{q}(x, a) d(\mathbb{S} \mu(x))<\infty .
$$

We can deal with the case $0<q<1$ similarly by replacing $l_{q}^{q}$ with $l_{q}$ :

$$
\begin{aligned}
\int_{X} d^{q}(x, a) d\left(\mathbb{S} \mu^{\omega}(x)\right) & =l_{q}\left(\sum_{i=1}^{N} p_{i}^{\omega} S_{i}^{\omega} \mu\left(\omega^{(i)}\right), \delta_{a}\right) \\
& =l_{q}\left(\sum_{i=1}^{N} p_{i}^{\omega} S_{i}^{\omega} \mu\left(\omega^{(i)}\right), \sum_{i=1}^{N} p_{i}^{\omega} S_{i}^{\omega} \delta_{b_{i}}\right) \\
& \leq \sum_{i=1}^{N} p_{i}^{\omega} r_{i}^{q} l_{q}\left(\mu\left(\omega^{(i)}\right), \delta_{b_{i}}\right)<\infty .
\end{aligned}
$$

To establish the contraction property, we consider $\mu, \nu \in \mathscr{E}_{q}$,

$$
\mu\left(\omega^{(i)}\right) \stackrel{d}{=} \mu(\omega), \quad v\left(\omega^{(i)}\right) \stackrel{d}{=} v(\omega), \quad i \in\{1,2, \ldots, N\},
$$

and $q \geq 1$. We have

$$
\begin{aligned}
F_{f(\mu), f(v)}(t) & =P\left(\left\{\omega \in \bar{\Omega} \mid l_{q}(f(\mu(\omega)), f(v(\omega)))<t\right\}\right) \\
& =P\left(\left\{\omega \in \bar{\Omega} \mid l_{q}\left(\sum_{i=1}^{N} p_{i}^{\omega} S_{i}^{\omega} \mu\left(\omega^{(i)}\right), \sum_{i=1}^{N} p_{i}^{\omega} S_{i}^{\omega} v\left(\omega^{(i)}\right)\right)<t\right\}\right) \\
& \geq P\left(\left\{\omega \in \bar{\Omega} \mid\left[\sum_{i=1}^{N} p_{i}^{\omega}\left(r_{i}\right)^{q} l_{q}^{q}\left(\mu\left(\omega^{(i)}\right), v\left(\omega^{(i)}\right)\right)\right]^{1 / q}<t\right\}\right) \\
& \geq P\left(\left\{\omega \in \bar{\Omega} \mid\left[\lambda_{q} l_{q}^{q}(\mu(\omega), v(\omega))\right]^{1 / q}<t\right\}\right)=F_{\mu, \nu}\left(\frac{t}{\lambda_{q}^{1 / q}}\right)
\end{aligned}
$$

for all $t>0$. 
In case $0<q<1$, one replaces $l_{q}^{q}$ everywhere by $l_{q}$ :

$$
\begin{aligned}
F_{f(\mu), f(v)}(t) & =P\left(\left\{\omega \in \bar{\Omega} \mid l_{q}(f(\mu(\omega)), f(v(\omega)))<t\right\}\right) \\
& =P\left(\left\{\omega \in \bar{\Omega} \mid l_{q}\left(\sum_{i=1}^{N} p_{i}^{\omega} S_{i}^{\omega} \mu\left(\omega^{(i)}\right), \sum_{i=1}^{N} p_{i}^{\omega} S_{i}^{\omega} v\left(\omega^{(i)}\right)\right)<t\right\}\right) \\
& \geq P\left(\left\{\omega \in \bar{\Omega} \mid\left[\sum_{i=1}^{N} p_{i}^{\omega}\left(r_{i}\right)^{q} l_{q}\left(\mu\left(\omega^{(i)}\right), v\left(\omega^{(i)}\right)\right)\right]^{1 / q}<t\right\}\right) \\
& \geq P\left(\left\{\omega \in \bar{\Omega} \mid\left[\lambda_{q} l_{q}(\mu(\omega), v(\omega))\right]<t\right\}\right)=F_{\mu, v}\left(\frac{t}{\lambda_{q}}\right)
\end{aligned}
$$

for all $t>0$. Thus $\mathbb{S}$ is a contraction map with ratio $\lambda_{q}^{1 / q \wedge 1}$. We can apply Corollary 3.5 for $r=\lambda_{q}^{1 / q \wedge 1}$. If $\mu^{*}$ is the unique fixed point of $\mathbb{S}$ and $\mu_{0} \in M_{q}$, then

$$
\begin{aligned}
& F_{\mathbb{S} n} \mu_{0}, \mu^{*}(t)=P\left(\left\{\omega \in \bar{\Omega} \mid l_{q}\left(\mathbb{S}^{n} \mu_{0}, \mu^{*}\right)<t\right\}\right) \\
& \geq P\left(\left\{\omega \in \bar{\Omega} \mid \frac{\lambda_{q}^{n / q}}{1-\lambda_{q}^{1 / q}} l_{q}\left(\mu_{0}, \mathbb{S} \mu_{0}\right)<t\right\}\right) \\
&=F_{\mu_{0}, \mathbb{S} \mu_{0}}\left(\frac{t\left(1-\lambda_{q}^{1 / q}\right)}{\lambda_{q}^{n / q}}\right), \\
& \lim _{n \rightarrow \infty} F_{\mathbb{S}^{n} \mu_{0}, \mu^{*}}(t)=1 \quad \forall t>0 .
\end{aligned}
$$

From $\mu_{n+1}(\omega)=\mathbb{S} \mu_{n}(\omega)$, it follows that $\mu_{n} \rightarrow \mu^{*}$ exponentially fast. Moreover, for $q \geq 1$,

$$
\sum_{i=1}^{\infty} \bar{P}\left(l_{q}^{q}\left(\mathbf{S}^{n} v_{0}, \mu^{*}\right) \geq \epsilon\right) \leq \sum_{i=1}^{\infty} \frac{e l_{q}^{q}\left(\mathbf{S}^{n} \mu_{0}, \mu^{*}\right)}{\epsilon} \leq c \sum_{i=1}^{\infty} \frac{\lambda_{q}^{n}}{\epsilon}<\infty .
$$

This implies by Borel-Cantelli lemma that $l_{q}\left(\mu_{n}, \mu^{*}\right) \rightarrow 0$ a.s.

For the uniqueness, let $\mathscr{D}$ be the set of probability distribution of members of $\mathscr{E}_{q}$. We define the probability metric on $\mathscr{D}$ by

$$
F_{\mathscr{A}, \mathscr{B}}(t)=\sup _{s<t} \sup \left\{F_{\mu, v}(s) \mid \mu \stackrel{d}{=} \mathscr{A}, v \stackrel{d}{=} \mathscr{B}\right\} .
$$

To establish the contraction property of $\mathscr{Y}$, we consider $\mathscr{A}, \mathscr{B} \in \mathscr{D}$. For $q \geq 1$, we get

$$
\begin{aligned}
F_{\mathscr{S} A, \mathscr{S} \mathscr{B}}(t) & =\sup _{s<t} \sup \left\{F_{\mathrm{S} \mu, \mathrm{S} v}(s) \mid \mu \stackrel{d}{=} \mathscr{A}, v \stackrel{d}{=} \mathscr{B}\right\} \\
& \geq \sup _{s<t} \sup \left\{F_{\mu, v}\left(\frac{s}{\lambda_{q}^{1 / q}}\right) \mid \mu \stackrel{d}{=} \mathscr{A}, v \stackrel{d}{=} \mathscr{B}\right\}=F_{\mathscr{A}, \mathscr{B}}\left(\frac{t}{\lambda_{q}^{1 / q}}\right)
\end{aligned}
$$

for all $t>0$. For $0<q<1$, the demonstration is similar. 
Consider $\mathscr{D}_{1}$ and $\mathscr{D}_{2}$ such that $\mathscr{S} \mathscr{D}_{1}=\mathscr{D}_{1}$ and $\mathscr{\mathscr { D }} \mathscr{D}_{2}=\mathscr{D}_{2}$.

Since $\mathscr{D}_{1}=\mathscr{S}^{n}\left(\mathscr{D}_{1}\right)$ and $\mathscr{D}_{2}=\mathscr{Y}^{n}\left(\mathscr{D}_{2}\right)$, we have

$$
F_{\mathscr{D}_{1}, \mathscr{D}_{2}}(t) \geq F_{\mathscr{D}_{1}, \mathscr{D}_{2}}\left(\frac{t}{r^{n}}\right)
$$

for all $t>0$. Using $\lim _{n \rightarrow \infty} r^{n}=0$, it follows that

$$
F_{\mathscr{D}_{1}, \mathscr{D}_{2}}(t)=1
$$

for all $t>0$.

REMARK 4.3. Since $\lambda_{q}^{1 / q} \rightarrow \max _{i} r_{i}$ as $q \rightarrow \infty$, we can regard [12, Theorem 4.2] as a limit case of Theorem 2.7. More precisely, if $\max _{i} r_{i}<1$, then $\operatorname{sprt} \mu^{*}$ is the unique compact set satisfying the random scaling law for sets $\left(S_{1}, \ldots, S_{N}\right)$.

ACKNOWLEDGMENT. This work was partially supported by Sapientia Foundation.

\section{REFERENCES}

[1] M. Arbeiter, Random recursive construction of self-similar fractal measures. The noncompact case, Probab. Theory Related Fields 88 (1991), no. 4, 497-520.

[2] M. F. Barnsley, Fractals Everywhere, Academic Press, Massachusetts, 1988.

[3] M. F. Barnsley and S. Demko, Iterated function systems and the global construction of fractals, Proc. Roy. Soc. London Ser. A 399 (1985), no. 1817, 243-275.

[4] Gh. Constantin and I. Istrăţescu, Elements of Probabilistic Analysis with Applications, Mathematics and Its Applications (East European Series), vol. 36, Kluwer Academic Publishers, Bucharest, 1989.

[5] K. J. Falconer, Random fractals, Math. Proc. Cambridge Philos. Soc. 100 (1986), no. 3, 559-582.

[6] S. Graf, Statistically self-similar fractals, Probab. Theory Related Fields 74 (1987), no. 3, 357-392.

[7] J. E. Hutchinson, Fractals and self-similarity, Indiana Univ. Math. J. 30 (1981), no. $5,713-747$.

[8] J. E. Hutchinson and L. Rüschendorf, Random fractal and probability metrics, Research Tech. Report MRR48, Australian National University, Canberra, 1998.

[9] , Random fractal measures via the contraction method, Indiana Univ. Math. J. 47 (1998), no. 2, 471-487.

[10] - Selfsimilar fractals and selfsimilar random fractals, Fractal Geometry and Stochastics, II (Greifswald, Koserow, 1998), Progr. Probab., vol. 46, Birkhäuser, Basel, 2000, pp. 109-123.

[11] J. Kolumbán and A. Soós, Invariant sets in Menger spaces, Studia Univ. BabeşBolyai Math. 43 (1998), no. 2, 39-48.

[12] J. Kolumbán and A. Soós, Invariant sets of random variables in complete metric spaces, Studia Univ. Babeş-Bolyai Math. 47 (2001), no. 3, 49-66.

[13] R. D. Mauldin and S. C. Williams, Random recursive constructions: asymptotic geometric and topological properties, Trans. Amer. Math. Soc. 295 (1986), no. 1, 325-346.

[14] K. Menger, Statistical metrics, Proc. Natl. Acad. Sci. USA 28 (1942), 535-537. 
[15] L. Olsen, Random Geometrically Graph Directed Self-Similar Multifractals, Pitman Research Notes in Mathematics Series, vol. 307, Longman Scientific \& Technical, Harlow, 1994.

[16] S. T. Rachev, Probability Metrics and the Stability of Stochastic Models, Wiley Series in Probability and Mathematical Statistics: Applied Probability and Statistics, John Wiley \& Sons, Chichester, 1991.

[17] B. Schweizer and A. Sklar, Statistical metric spaces, Pacific J. Math. 10 (1960), 313-334.

[18] _ Probabilistic Metric Spaces, North-Holland Series in Probability and Applied Mathematics, North-Holland Publishing, New York, 1983.

[19] V. M. Sehgal, A fixed point theorem for mappings with a contractive iterate, Proc. Amer. Math. Soc. 23 (1969), 631-634.

[20] H. Sherwood, On E-spaces and their relation to other classes of probabilistic metric spaces, J. London Math. Soc. (2) 44 (1969), 441-448.

[21] B. Z. Vulikh, A Brief Course in the Theory of Functions of a Real Variable, Mir Publishers, Moscow, 1976.

József Kolumbán: Faculty of Mathematics and Computer Science, Babes-Bolyai University, 3400 Cluj-Napoca, Romania

E-mail address: kolumban@math.ubbcluj.ro

Anna Soós: Faculty of Mathematics and Computer Science, Babes-Bolyai University, 3400 Cluj-Napoca, Romania

E-mail address: asoos@math.ubbcluj .ro

Ibolya Varga: Faculty of Mathematics and Computer Science, Babes-Bolyai University, 3400 Cluj-Napoca, Romania

E-mail address: ivarga@cs . ubbcluj . ro 


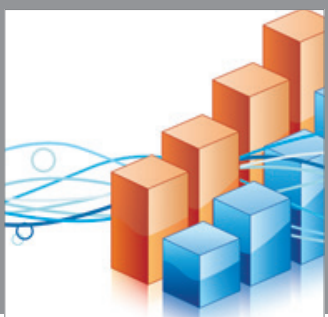

Advances in

Operations Research

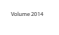

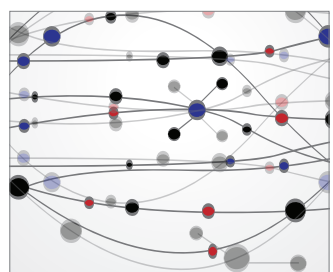

\section{The Scientific} World Journal
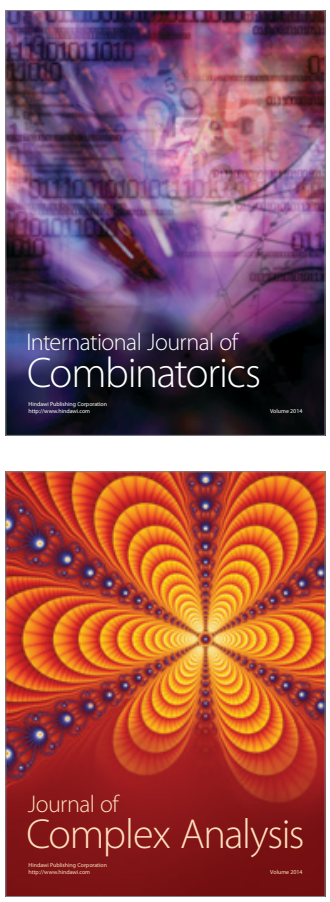

International Journal of

Mathematics and

Mathematical

Sciences
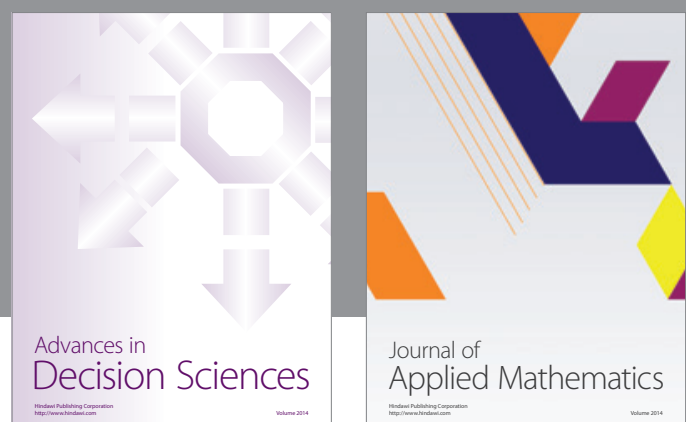

Journal of

Applied Mathematics
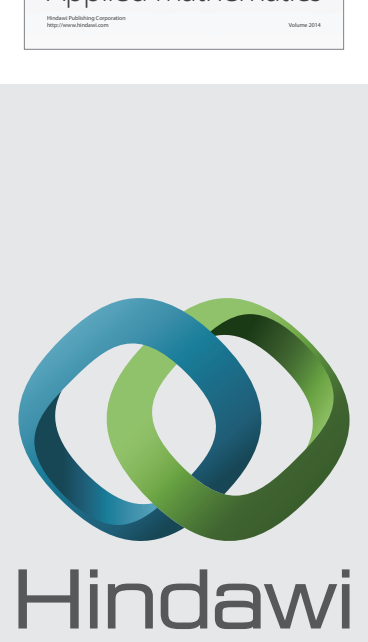

Submit your manuscripts at http://www.hindawi.com
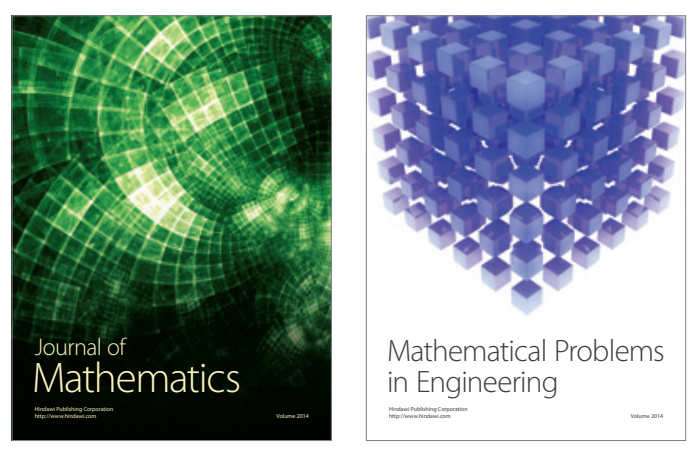

Mathematical Problems in Engineering
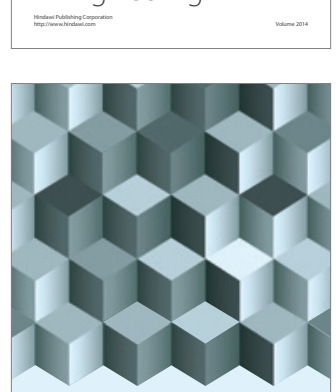

Journal of

Function Spaces
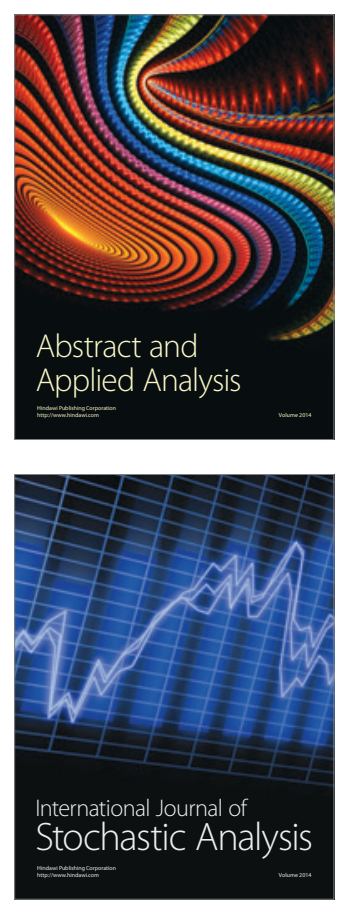

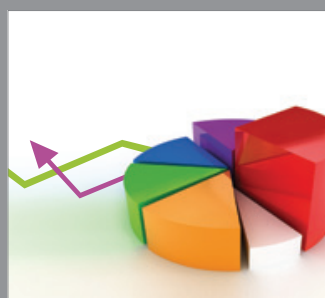

ournal of

Probability and Statistics

Promensencen
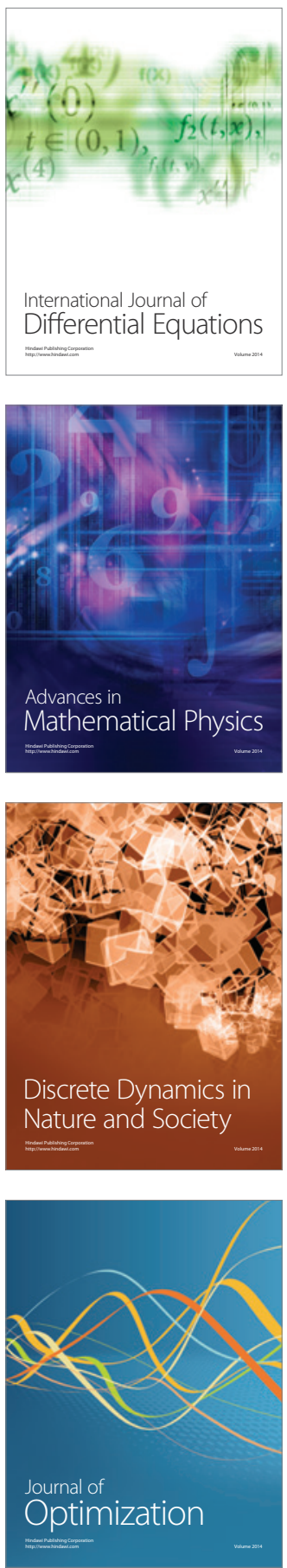